\title{
VALIDAÇÃO DE MÉTODO PARA A DETERMINAÇÃO DE 5-HIDROXIMETILFURFURAL EM MEL POR CROMATOGRAFIA LÍQUIDA E SUA INFLUÊNCIA NA QUALIDADE DO PRODUTO
}

\author{
Gisele da Silveira Lemos*, José Soares dos Santos e Maria Lúcia Pires dos Santos \\ Departamento de Ciências Naturais, Universidade Estadual do Sudoeste da Bahia, CP 95, 45083-900 Vitória da Conquista - BA, Brasil
}

Recebido em 16/11/09; aceito em 9/4/10; publicado na web em 24/8/10

\begin{abstract}
METHOD VALIDATION TO HMF DETERMINATION IN HONEY BY HPLC-UV AND ITS INFLUENCE ON THE PRODUCT QUALITY. In honey 5-hydroxymethyl-2-furaldehyde (HMF) is one of the most typical products of degradation: it is usually absent in fresh honey, but its concentration tends to rise as a result of heating processes or long-term storage. The validation protocol was performed in terms of detection and quantification limits, precision (by repeatability and intermediate precision), linearity and accuracy (by recovery tests). The method has been tested on 15 honey samples of different ages and geographical origin. HMF correlated highly with the age of the samples has been considered a very important parameter to put these honeys on the market or not and/or to estimate their shelf life.
\end{abstract}

Keywords: HMF; honey; HPLC-UV.

\section{INTRODUÇÃO}

O mel é um alimento natural produzido por abelhas melíferas, a partir do néctar ou secreções de plantas, modificado e armazenado em colmeias. ${ }^{1}$ É um produto semilíquido que contém uma mistura complexa de carboidratos (principalmente glicose e frutose), ácidos orgânicos, lactonas, aminoácidos, minerais, vitaminas, enzimas, pólen, cera e pigmentos. ${ }^{2}$

O crescente consumo do mel nas últimas décadas, estimulado pelas propriedades reconhecidamente benéficas à saúde humana, vem exigindo um controle de qualidade cada vez mais eficiente deste produto. Os mais importantes parâmetros para avaliar a qualidade do mel são teores de contaminantes (antibióticos, pesticidas, metais pesados), origem floral, área de produção e tempo de prateleira. $\mathrm{O}$ parâmetro mais utilizado para conferir se ocorreu um aquecimento indesejável, ou avaliar o tempo de estocagem do mel, são os teores de 5-hidroximetilfurfural (HMF) e o índice de diastase. ${ }^{3}$

O HMF geralmente não está presente em méis frescos e seu conteúdo tende a aumentar durante o processo de aquecimento e o longo tempo de estocagem, ${ }^{4-6}$ pela reação de Maillard em carboidratos ou desidratação catalítica ácida das hexoses. O HMF é um dos principais produtos de degradação no mel sendo o aumento de sua concentração influenciada pelo baixo $\mathrm{pH}$, acidez total, minerais, origem botânica, umidade, temperatura e stress fotoquímico. ${ }^{7}$

O Codex Alimentarius e a União Europeia estabeleceram um nível máximo de HMF em mel de $40 \mathrm{mg} \mathrm{kg}^{-1}$, com exceção para os méis oriundos de países de temperaturas tropicais e para os méis com baixos níveis enzimáticos, cujos níveis máximos de HMF estabelecidos são de 80 e $15 \mathrm{mg} \mathrm{kg}^{-1}$, respectivamente. ${ }^{7,8}$

A flutuação de HMF em méis uniflorais armazenados à temperatura ambiente tem sido considerado como um parâmetro muito importante para certificar a qualidade dos méis disponíveis no mercado ou estimar sua estabilidade de prateleira. ${ }^{3}$

A presença de componentes potencialmente tóxicos (tais como aldeídos reativos) em alimentos vem aumentando a atenção de agências protetoras do consumidor e de controle de qualidade. Em particular, alguns estudos mostram que o HMF está entre as substâncias de risco de citotoxicidade, genotoxicidade e atividade mutagênica. ${ }^{9}$ Existem vários métodos propostos para determi-

\footnotetext{
*e-mail: gisalemos@ig.com.br
}

nar o HMF em mel, espectrofotometricamente pelo método de Winkler ou modificações desse método e o método de White que envolvem medidas de absorvância na região ultravioleta (UV) e por cromatografia líquida de alta eficiência (CLAE). Este último parece ser o mais apropriado devido a interferências provocadas por substâncias derivadas, provavelmente, do aquecimento ou estocagem inadequada quando utiliza os métodos UV. Além disso, a utilização de CLAE proporciona análise de outros componentes. ${ }^{4,10}$ O crescimento da atenção que a comunidade científica vem dando às potencialidades dos efeitos tóxicos do HMF requer esforços para estabelecer um método rápido e sensível para determinar o analito em matrizes reais. ${ }^{7}$

Os objetivos deste trabalho foram otimizar e validar um método que utiliza cromatografia líquida de alta eficiência (CLAE) para determinação do HMF em amostras de méis provenientes do Sudoeste da Bahia e, avaliar a influência do tempo de estocagem na concentração do HMF nos méis produzidos no Sudoeste da Bahia, visando uma melhor eficiência no controle de qualidade do produto comercializado.

\section{PARTE EXPERIMENTAL}

\section{Amostras de mel}

Foram avaliadas 15 amostras de méis de abelhas Apis mellifera oriundas de diferentes municípios localizados na região Sudoeste do estado da Bahia, incluindo diferentes origens botânicas, características de solo e clima, de acordo com a Tabela 1. As amostras foram obtidas diretamente em associações de produtores e na casa do mel da escola de Agronomia da Universidade Estadual do Sudoeste da Bahia.

As amostras foram armazenadas em frascos de polipropileno contendo 250 e $500 \mathrm{~g}$ de mel, mantidas à temperatura ambiente em local fresco e escuro antes das análises.

\section{Reagentes e soluções}

Todas as soluções foram preparadas utilizando água ultrapura, obtida pelo sistema de purificação Millipore (Millipore, SP, Brasil). A fase móvel utilizada foi composta de água ultrapura e acetonitrila com grau HPLC (J. T. Baker). A fase móvel foi filtrada através de uma membrana de acetato de celulose $0,45 \mu \mathrm{m}$ (Millipore) para remover todas as impurezas. 
Tabela 1. Concentração média de HMF em $\left(\mathrm{mg} \mathrm{kg}^{-1} \pm\right.$ desvio padrão) das amostras de méis por ano/mês de coleta em diferentes municípios do Estado da Bahia

\begin{tabular}{lccc}
\hline Ano de coleta & Mês & Município & HMF $\left(\mathrm{mg} \mathrm{kg}^{-1}\right)$ \\
\hline 1999 & ---- & Vitória da Conquista & $400,8 \pm 15,5$ \\
2004 & ---- & Vitória da Conquista & $267,4 \pm 21,2$ \\
2006 & Junho & Vitória da Conquista & $116,9 \pm 10,2$ \\
& Agosto & Itabela & $180,5 \pm 7,6$ \\
2007 & Abril & Mortugaba & $73,80 \pm 4,50$ \\
& Agosto & Seabra & $25,45 \pm 1,14$ \\
2008 & Outubro & Jequié & $76,80 \pm 2,15$ \\
& Fevereiro & Amargosa & $66,60 \pm 2,20$ \\
& Março & Itambé & $<\mathrm{LD}$ \\
& Março & Tremedal & $27,40 \pm 0,60$ \\
& Julho & Vitória da Conquista & $26,80 \pm 0,25$ \\
& Dezembro & Maracás & $28,70 \pm 0,25$ \\
& Fevereiro & Vitória da Conquista & $26,90 \pm 0,55$ \\
& Março & Vitória da Conquista & $26,73 \pm 1,55$ \\
\hline & Abril & Maracás &
\end{tabular}

As soluções analíticas de referência foram preparadas por sucessivas diluições de uma solução $5 \mathrm{~g} \mathrm{~L}^{-1}$ de HMF com 99,95\% de pureza (MFS, Advantec).

Todas as soluções foram armazenadas em frascos de polipropileno descontaminados (Company, Rochester).

\section{Preparo das amostras}

Todas as vidrarias e frascos de polipropileno foram limpos com uma solução de detergente neutro e ácido nítrico $10 \%$ (v/v) por $24 \mathrm{~h}$.

Precisamente $5 \mathrm{~g}$ de cada amostra de mel foram diluídas em água ultrapura, em um balão volumétrico de $50,00 \mathrm{~mL}$. A seguir, foram filtradas em membrana borossilicato de $0,45 \mu \mathrm{m}$ e o filtrado recolhido em frasco de polietileno.

As amostras de mel foram separadas em 3 grupos de acordo com o tempo de estocagem, sendo 5 amostras com tempo inferior/ igual a 12 meses, 5 amostras entre 12 e 24 meses e as outras 5 com tempo superior/igual a 24 meses, conforme apresentado na Tabela 1.

\section{Equipamento e condições cromatográficas}

O sistema de cromatografia líquida CLAE utilizado é composto de uma bomba series 200, válvula de amostragem, loop de $20 \mu \mathrm{L}$ e um detector Series 200 UV/VIS, Perkin Elmer (USA). A separação do analito foi realizada utilizando-se uma coluna RP-C18 $(250 \mathrm{~mm}$ x 4,6 mm, $5 \mu \mathrm{m}$ de tamanho de partícula, Thermo, USA). O volume de amostra injetada foi de $20 \mu \mathrm{L}$, a qual foi submetida a uma eluição isocrática com água/acetonitrila (80:20) e fluxo de $1 \mathrm{~mL} \mathrm{~min}{ }^{-1}$, e a detecção do analito realizada em $285 \mathrm{~nm}$.

\section{Validação do método}

A validação do método de determinação do HMF em méis por CLAE foi realizada com base na análise dos seguintes parâmetros: linearidade, limite de detecção e quantificação, exatidão (recuperação), precisão (repetibilidade: precisão intradia e precisão intermediária - interdias), e robustez.

Todas as operações matemáticas e estatísticas foram realizadas com auxílio dos programas Origin 5.0 e Statistica 7.0 (Statistica for Windows, Statsoft, Inc, 1999. Tulsa, OK, EUA).

\section{RESULTADOS E DISCUSSÃO}

\section{Linearidade}

A curva de calibração foi construída utilizando a padronização externa, a partir de 5 pontos das soluções padrão de HMF em água nas concentrações de 10 a $50 \mathrm{mg} \mathrm{L}^{-1}$, preparadas no mesmo dia em que se realizaram as análises. Os padrões foram preparados em triplicata e cada uma das replicatas foi injetada 5 vezes. A linearidade foi avaliada entre dias diferentes e foi estimada pela análise de regressão linear através do método dos mínimos quadrados.

As curvas analíticas traçadas por padronização externa para HMF demonstraram excelente linearidade na faixa de 10 a $50 \mathrm{mg} \mathrm{L}^{-1}$, com coeficiente de correlação médio de 0,99899 . Isto indica a validade do método, pois obedece a uma correlação linear nos intervalos de concentração avaliados. A curva de calibração obtida pelo método dos mínimos quadrados pode ser expressa pela equação $y=a+b x$, sendo os valores encontrados dispostos na Tabela 2 .

Tabela 2. Equação da reta, coeficiente de correlação (r), limites de quantificação (LQ) e detecção (LD) obtidos no método de validação para HMF por CLAE-UV

\begin{tabular}{lccc}
\hline Equação da reta & $\mathrm{r}$ & $\mathrm{LQ}\left(\mathrm{mg} \mathrm{L}^{-1}\right)$ & $\mathrm{LD}\left(\mathrm{mg} \mathrm{L}^{-1}\right)$ \\
\hline $\mathrm{y}=-39,8295+25,2102 \mathrm{x}$ & 0,9986 & 0,17 & 0,05 \\
\hline
\end{tabular}

O processo de separação e determinação do HMF proposto apresentou uma boa seletividade. Isso se deve à inexistência de interferentes nas amostras de mel em estudo. Como pode ser observado na Figura 1, o tempo retenção do HMF nos padrões e nas amostras foi similar (3,77 e 3,85 min, respectivamente).

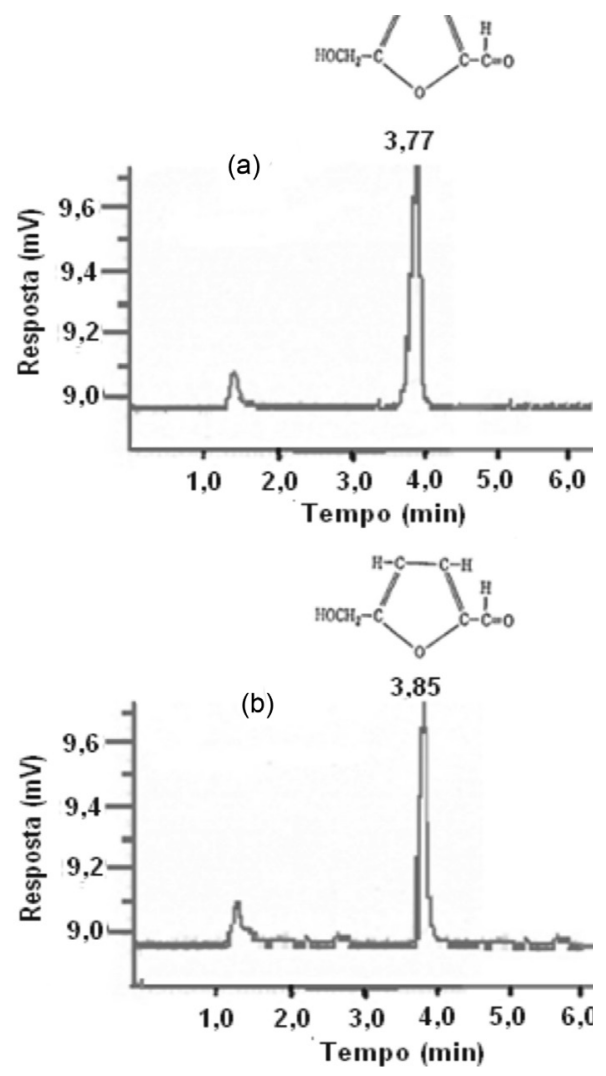

Figura 1. Cromatogramas obtidos pela análise de soluções de HMF $40 \mathrm{mg}$ $\mathrm{kg}^{-1}$ (a) preparada em água, (b) preparada na amostra 


\section{Limites de detecção e de quantificação}

Os termos limite de quantificação e limite de detecção são utilizados para demonstrar a habilidade do método em quantificar/detectar baixas concentrações de um analito. ${ }^{11-14}$

O limite de detecção é definido como a menor concentração de um analito que o método é capaz de diferenciar do sinal ruído. ${ }^{11,15}$ $\mathrm{O}$ limite de quantificação é definido como a menor concentração do analito de interesse em uma amostra, que pode ser quantitativamente determinado com valores aceitáveis de precisão e exatidão. ${ }^{11-14}$

O limite de detecção foi definido como três vezes o ruído da linha de base, obtido pela determinação de diluições sucessivas e decrescentes da solução padrão de menor concentração. O limite de quantificação também foi determinado através do ruído da linha de base. Foi considerado como limite de quantificação a concentração determinada a partir da relação sinal ruído superior a 10:1. ${ }^{11,16}$

Os valores dos limites de detecção e de quantificação obtidos encontram-se na Tabela 1 e foram avaliados para determinação da sensibilidade. Esses resultados demonstram que o método é sensível para detectar e quantificar os níveis de concentração HMF presentes em amostras de méis.

\section{Exatidão (Recuperação)}

A exatidão foi avaliada em termos da recuperação. A recuperação avalia a eficiência do método de tratamento das amostras, extração, expressa como a porcentagem da quantidade conhecida de um analito. A porcentagem de recuperação foi obtida pelo valor da relação entre a concentração média determinada experimentalmente e a concentração teórica correspondente após a adição do analito, multiplicada por 100. ${ }^{11-15}$

O método de adição padrão foi utilizado, pois não é possível obter a matriz isenta do analito. Para isso, quantidades conhecidas de HMF foram adicionadas em triplicata no mel em três níveis de concentração, 10, 30 e $50 \mathrm{mg} \mathrm{L}^{-1}$, e em seguida manipuladas conforme procedimento de preparo da amostra. As amostras sem adição de padrão e com adição de padrão foram analisadas simultaneamente, e a recuperação expressa em porcentagem da quantidade medida da substância em relação à quantidade adicionada na matriz.

A exatidão do método foi determinada por meio da obtenção da porcentagem de recuperação média avaliada em três níveis de concentração, em triplicata. Os valores obtidos encontram-se descritos na Tabela 2. A determinação da recuperação do analito foi realizada pela sua quantificação na matriz em estudo pelo emprego de um padrão analítico. O grau de recuperação variou entre 90 a $102 \%$, o que está dentro das especificações esperadas para matrizes complexas. ${ }^{16}$

\section{Precisão}

A precisão de um método analítico é a medida dos erros aleatórios e representa a proximidade dos resultados obtidos a partir de medidas independentes de amostragens múltiplas de uma amostra homogênea. A precisão do método foi avaliada levando em consideração a repetibilidade e a precisão intermediária. ${ }^{11-15}$

\section{Repetibilidade (precisão intradia)}

Define a precisão do método em repetir, em um curto intervalo de tempo, os resultados obtidos nas mesmas condições de análise, ou seja, com o mesmo analista, mesmo equipamento, mesmo laboratório e utilizando os mesmos reagentes. ${ }^{11-15}$

Para avaliar a repetibilidade analítica do método empregada na análise, prepararam-se 9 amostras, contemplando o intervalo linear do método, ou seja, 3 níveis de concentração (10, 30 e $\left.50 \mathrm{mg} \mathrm{L}^{-1}\right)$, com 3 replicatas cada uma. Essas foram injetadas em quintuplicata no mesmo dia, nas mesmas condições cromatográficas e pelo mesmo analista. As respostas foram expressas como o desvio padrão relativo (DPR).

\section{Precisão intermediária (interdias)}

Define a habilidade do método em fornecer os mesmos resultados quando as análises são conduzidas no mesmo laboratório, mas em dias diferentes. Para a determinação da precisão intermediária, recomenda-se um mínimo de 2 dias diferentes com analistas diferentes. ${ }^{11-14}$ Foram preparadas 15 amostras, avaliadas em 2 diferentes dias. As amostras foram injetadas em quintuplicata e os resultados expressos como desvio padrão relativos.

Os resultados da repetibilidade e precisão intermediária encontram-se na Tabela 3, como desvio padrão relativo. Os valores do desvio padrão relativo obtidos das medidas estão entre 0,42 a 5,38 indicando uma precisão aceitável para a validação de um método analítico, uma vez que valores em torno de $15 \%$ são considerados admissíveis de acordo com a literatura. ${ }^{17}$

Tabela 3. Repetibilidade, precisão intermediária e exatidão obtidas no método de validação para HMF por CLAE-UV

\begin{tabular}{lccc}
\hline $\begin{array}{l}\text { Concentração } \\
\left(\mathrm{mg} \mathrm{L}^{-1}\right)\end{array}$ & $\begin{array}{c}\text { Repetibilidade } \\
\left(\% \mathrm{DPR} .^{*}\right)\end{array}$ & $\begin{array}{c}\text { Precisão } \\
\text { intermediária }\left(\% \mathrm{DPR}^{*}\right)\end{array}$ & $\begin{array}{c}\text { Exatidão/ } \\
\text { Recuperação }(\%)\end{array}$ \\
\hline 10 & 0,42 & 3,31 & 102,1 \\
20 & - & 3,13 & - \\
30 & 5,38 & 2,19 & 94,30 \\
40 & - & 3,51 & - \\
50 & 1,88 & 5,88 & 104,98 \\
\hline
\end{tabular}

\section{Robustez}

A robustez de um método analítico mede sua suscetibilidade frente a pequenas variações que podem ocorrer durante as análises de rotina. ${ }^{11,12,14}$

A robustez do método proposto foi avaliada em um planejamento fatorial em dois níveis $2^{2}$, de acordo com Tabela 4. Os parâmetros avaliados foram fluxo da fase móvel e concentração de acetonitrila na fase móvel. Os níveis inferiores e superiores para as variações dos parâmetros experimentais ficaram compreendidos entre $2 \%$ para o fluxo e $5 \%$ para a concentração de acetonitrila na fase móvel. Todos os experimentos foram desenvolvidos em duplicata, e no ponto central foram realizados experimentos em triplicata. ${ }^{18}$

Tabela 4. Matriz de planejamento fatorial $2^{2}$ para avaliação da robustez

\begin{tabular}{lcc}
\hline Amostra & Vazão $\left(\mathrm{mL} \mathrm{min}^{-1}\right)$ & Acetonitrila $(\%)$ \\
\hline 1 & + & + \\
2 & + & - \\
3 & - & + \\
4 & - & - \\
5 & 0 & 0 \\
6 & 0 & 0 \\
7 & 0 & 0 \\
\hline
\end{tabular}

A significância dos efeitos das variáveis e das possíveis interações entre elas foi checada com base no gráfico de Pareto, Figura 2. Segundo o gráfico de Pareto, tem-se que as variáveis analisadas foram robustas nas variações estudadas.

\section{Influência do tempo de estocagem na qualidade do mel}

Existe no Brasil uma legislação específica para o mel, que 
(a)

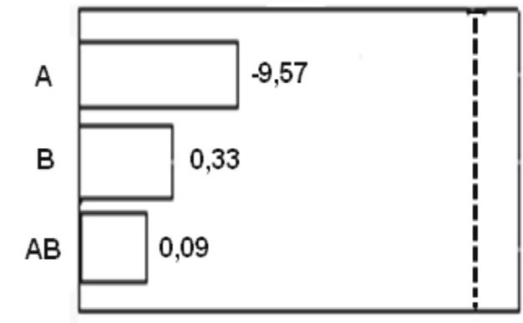

(b)

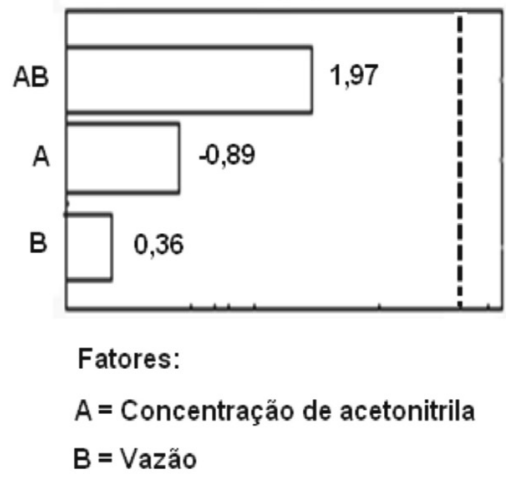

Figura 2. Gráfico de Pareto para os efeitos padronizados em p=0,05: (a) resposta em função do tempo de retenção ( $\mathrm{min})$, (b) resposta em função da altura do pico $(\mathrm{mV})$

estabelece parâmetros de controle de qualidade para o produto. A Portaria 367/97, da Secretaria do Ministério da Agricultura, Pecuária e Abastecimento, que regulamenta a identidade e a qualidade do mel, estabelece uma concentração máxima para o HMF de $60 \mathrm{mg} \mathrm{kg}^{-1}{ }^{19}$

$\mathrm{O}$ método mostrou-se eficaz para a determinação do HMF nas amostras de mel oriundas do Sudoeste da Bahia. De acordo com os dados apresentados na Figura 3 e Tabela 1, pode-se verificar que os valores das concentrações do HMF aumentam com o envelhecimento do mel. Todas as amostras produzidas no período de 1999 a 2006 apresentaram concentrações do HMF acima do estabelecido pela Portaria 367/97 do Ministério da Agricultura, Pecuária e Abastecimento. O mesmo foi observado em duas das três amostras avaliadas do ano 2007 e em uma das cinco amostras do ano de 2008.

Por outro lado, todas as amostras de mel produzidos em 2009 apresentaram níveis de HMF dentro dos padrões de qualidade estabelecidos. Observou-se, também, que as concentrações de HMF nessas amostras estão de acordo com os valores publicados na literatura para

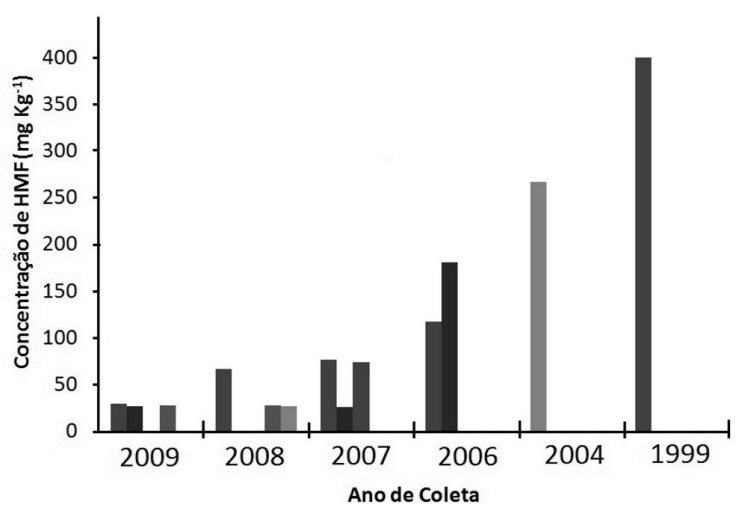

Figura 3. Concentração $\left(\mathrm{mg} \mathrm{kg}^{-1}\right)$ de HMF em função do tempo de estocagem das amostras de méis méis brasileiros. ${ }^{20,21}$ Desta forma, a determinação da concentração do HMF em méis estocados pode ser utilizada para avaliar a estabilidade e o prazo de validade do produto comercializado, em virtude dos possíveis efeitos tóxicos do HMF e seus derivados.

\section{CONCLUSÕES}

Um método rápido e direto, que utilizou CLAE em fase reversa, foi otimizado e validado para determinação de HMF em amostras de mel oriundas do Sudoeste da Bahia. O método foi testado para 15 amostras de mel com diferentes tempos de estocagem e origem geográfica. Das 15 amostras avaliadas, 6 apresentaram valores conforme recomendado pela legislação brasileira $\left(60 \mathrm{mg} \mathrm{kg}^{-1}\right)$, das quais somente uma dessas amostras foi estocada por um tempo superior a 12 meses. A correlação entre aumento na concentração de HMF com o aumento no tempo de estocagem do mel sugere perda de qualidade com o envelhecimento e possível risco à saúde dos consumidores. Finalmente, os parâmetros de validação demonstraram que o método apresentou baixos valores de LD e LQ $\left(0,05\right.$ e $0,17 \mathrm{mg} \mathrm{kg}^{-1}$, respectivamente), boas precisão e exatidão, constituindo-se em uma importante ferramenta na avaliação de qualidade dos méis estocados.

\section{REFERÊNCIAS}

1. Gidamis, A.; Chove, B.; Shayo, N.; Nnko, S.; Bangu, N.; Plant Foods for Human Nutrition 2004, 59, 129.

2. Zapala, M.; Fallico, B.; Arena, E.; Verzera, A.; Food Chem. 2004, 85, 305.

3. Fallico, B.; Arena, E.; Zappala, M.; J. Food Sci. 2008, 73, 625.

4. Zapala, M.; Falico, B.; Arena, E.; Verzera, A.; Food Control 2005, 16, 273.

5. Risner, C. H.; Kiser, M. J.; Dube, M. F.; J. Food Sci. 2006, 71, 179.

6. Gokmen, V.; Acar, J.; J. Chromatogr., A 1999, 847, 69.

7. Spano, N.; Casula, L.; Panzanelli, A.; Pilo, M. I.; Piu, P. C.; Scanu, R.; Tapparo, A.; Sanna, G.; Talanta 2006, 68, 1390.

8. Coco, F. L.; Valentini, C.; Novelli, V.; Ceccon, L.; J. Chromatogr., A 1996, 749, 95 .

9. Spano, N.; Ciulua, M.; Floris, I.; Panzanellia, A.; Pilo, M. I.; Piua, P. C.; Salis, S.; Sanna, G.; Talanta 2009, 78, 310.

10. Nozal, M. J.; Bernal, J. L.; Toribio, L.; Jimenez, J. J.; Martín, M. T.; J. Chromatogr., A 2001, 917, 95.

11. Cassiano, N. M.; Barreiro, J. C.; Martins, L. R. R., Oliveira, R. V.; Cass, Q. B.; Quim. Nova 2009, 32, 1021.

12. Ribani, M.; Bottoli, C. B. G.; Collins, C. H.; Jardim, I. C. S. F.; Melo, L. F. C.; Quim. Nova 2004, 27, 771.

13. Beck, R. C. R.; Cardoso, S. G.; Athayde, M. L.; Codevilla, C.; Oliveira, F. K.; Dalmora, S. L.; Quim. Nova 2007, 30, 1225.

14. Friedrich, R. B.; Ravanello, A.; Cichota, L. C.; Rolim, C. M. B.; Beck, R. C. R.; Quim. Nova 2009, 32, 1052.

15. Agência Nacional de Vigilância Sanitária (ANVISA); Resolução 899, de 29/05/2003, que trata da validação de métodos analíticos e bioanalíticos, Brasília, 2003.

16. Celeghini, R. M. S.; Sousa, I. M. O.; Silva, A. P.; Rodrigues, R. A. F.; Foglio, M. A.; Quim. Nova 2009, 32, 875.

17. Aquino, F. W. B.; Amorin, A. G. N.; Prata, L. F.; Nascimento, R. F.; Ciênc. Tecnol. Aliment. 2004, 24, 32.

18. Costa, L. M.; Korn, M. G. A.; Castro, J. T.; Santos, W. P. C.; Carvalho, E. V.; Nogueira, A. R. A.; Quim. Nova 2006, 29, 149.

19. http://www.agridat.mq.gov/mercosul/queijosmeilmetportmei.html, acessada em Março 2007.

20. Marchini, L. C.; Moreti, A. C. C. C., Otsuk, I. P.; Ciênc. Tecnol. Aliment. 2005, 25, 8.

21. Rodrigues, A. E.; Silva, E. M. S.; Beserra, E. M. F.; Rodrigues, M. L.; Ciência Rural 2005, 35, 1166. 